\title{
ESTADO ACTUAL DE LA DIVERSIDAD FLORÍSTICA DEL PÁRAMO SECTORES: EL ESPINO Y PALAMBE, SALLIQUE, JAÉN. CAJAMARCA. PERÚ
}

\section{ACTUAL STATUS OF THE FLORISTIC DIVERSITY OF THE PARAMO: EL ESPINO AND PALAMBE SECTORS, SALLIQUE, JAÉN. CAJAMARCA. PERÚ}

\author{
José Luís Marcelo Peña ${ }^{1}$, Isidoro Sánchez Vega ${ }^{2}$ y José F. Millán Tapia ${ }^{3}$
}

\section{Resumen}

Se presentan los resultados de un estudio realizado al Norte de la Depresión Huancabamba, en los páramos de El Espino y Palambe, Jaén, Cajamarca - Perú. Se reportan un total de 252 especies de 130 géneros pertenecientes a 58 familias. Se encontraron tres nuevos géneros y 40 especies para el páramo del Neotrópico. La diversidad florística es rica en la zona, con respecto a otros páramos del Perú y Sur del Ecuador, que por sus niveles de endemismos califica como prioritaria para conservación.

Palabras claves: Páramos, Cajamarca, Jaén, diversidad florística, especies endémicas, flora, conservación

\begin{abstract}
The present work shows the results from a study performed north of the Huancabamba Depression, at El Espino and Palambe Paramos, located in Jaen, Cajamarca - Peru. A total of 252 species from 130 genera belonging to 58 families are reported in this work. 3 new genera and 40 species for the Neotropical paramo have been found. This zone is rich in floristic diversity, compared to other paramos in Peru and southern Ecuador, and due to its endemism levels it qualifies as a priority area for conservation purposes.
\end{abstract}

Key words: Páramos, Cajamarca, Jaén, diversidad florística, especies endémicas, flora, conservación

\section{Introducción}

En el Perú el concepto de páramos del Norte o jalca ha sido utilizado por Weberbauer (1945), para describir la región de pajonal graminoso carente de árboles que se extiende entre $8^{\circ} 30^{\prime}-6^{\circ} 30^{\prime}$ Latitud Sur, al oeste del río Marañón; sin embargo, Luteyn (1999) indica que el páramo es un ecosistema Neotropical ubicado entre el límite del bosque cerrado y las nieves perpetuas, se localiza a lo largo de las cordilleras o en picos aislados a altitudes que oscilan entre los 3000 - $5000 \mathrm{~m}$ aproximadamente; se concentra en la parte noroeste de Sudamérica, principalmente en Venezuela, Colombia Ecuador, con algunos relictos en Costa Rica, Panamá y el Norte de Perú, entre los $11^{\circ} \mathrm{N}-8^{\circ} \mathrm{S}$ de L.S.

El páramo del Perú, es un ecosistema que se caracteriza por presentar un paisaje dominado por gramíneas macollantes cespitosas de hojas convolutas, plantas en rosetas con escapos emergentes, rosetas acaules, arbustos siempre verdes de hojas coriáceas o pubescentes y plantas almohadilladas; es muy húmedo, presenta 85 - 95\% de cobertura vegetal y una alta diversidad florística. Este tipo de vegetación se encuentra dispersa a lo largo de las cadenas de montañas altas desde los 3000 a $3700 \mathrm{~m}$ al Norte de la Depresión de Huancabamba (Marcelo \& Millán, 2004)

El páramo en realidad posee una gran variedad vegetal mucho mayor de lo que se define como "lugar yermo desprovisto de árboles” (Luteyn, 1999). El mismo autor indica que el páramo en toda su extensión en el Neotrópico cubre alrededor del 2\% de la superficie de los países; sin embargo, presenta cerca de 3400 especies de plantas vasculares de 500 géneros pertenecientes a 125 familias.

La desaparición de los páramos está asociada a la minería, la deforestación de los bosques naturales, la quema y otras prácticas inapropiadas que provocan su destrucción y en consecuencia la erosión de suelos (Rodríguez, 1996). Marcelo \& Millán (2004) indican que la actividad pecuaria genera severos impactos sobre la vegetación, ya que cada dos o tres años se queman los pajonales para obtener retoños frescos y asegurar la alimentación del ganado vacuno; así también, la extracción de plantas medicinales está reduciendo significativamente las poblaciones de las siguientes especies vegetales: "cóndor blanco" Huperzia macbride, "cóndor amarillo" $H$. weberbaueri, “cóndor verde” $H$. brevifolia y "cóndor" H. capellae (Lycopodiaceae); "valeriana” Phyllactis rigida (Valerianiacea), "trencilla verde” Lycopodium jussiaei, L. thyoides, L. vestibum (Lycopodiaceae), "trencilla negra” Jamesonia alstonii, J. goudotiii (Pteridaceae), “calaguala” Blechnum loxense, B. auratum (Blechnaceae), "rabo de zorro” Alchemilla verticilada (Rosaceae), “carqueja” Baccharis genistelloides (Asteraceae), poniendo en peligro su 


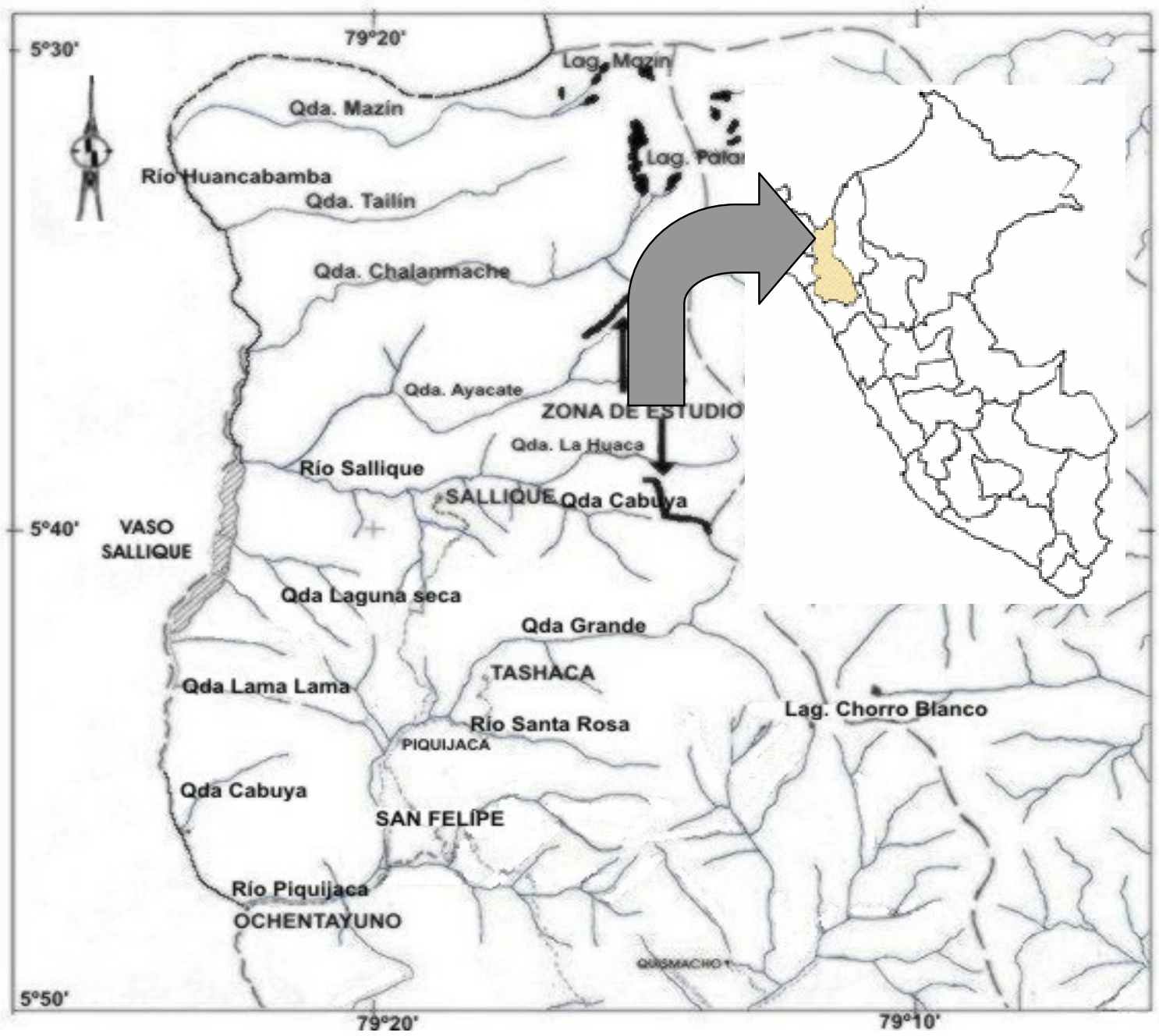

Figura 1. Localización de la zona de estudio

capacidad de regeneración, procesos que están acelerando la pérdida de la biodiversidad de nuestro patrimonio natural (Marcelo \& Millán, 2004).

En este contexto, los estudios que proporcionan información para mejorar el conocimiento de este ecosistema son muy escasos; por lo tanto, comprendiendo la urgente necesidad de estudiar y conocer el patrimonio vegetal de nuestro país, este trabajo contribuirá al conocimiento de la diversidad florística de una parte de los páramos del Norte del Perú.

\section{Materiales y métodos}

Ubicación

El área de estudio se encuentra geográficamente al Norte de la depresión Huancabamba, y al Este del río del mismo nombre, en los páramos del sector El Espino y Palambe, Jaén, Cajamarca - Perú entre las coordenadas $05^{\circ} 35^{\prime}-05^{\circ} 41^{\prime}$ L.S. y $79^{\circ} 15^{\prime}-79^{\circ} 16^{\prime}$ L.O. a altitudes entre 3000-3560 m (Figura 1).

\section{Caracterización ecológica}

Según INRENA (1994), el área de estudio presenta dos zonas de vida: bosque húmedo - Montano Bajo Tropical (bh-MBT) y bosque muy húmedo - Montano Tropical (bmh-MT). El primero entre los 2800 y 3200 msnm y la segunda entre los 3200 y los $3500 \mathrm{~m}$. El clima es frío y húmedo, con temperaturas promedio es de $6^{\circ}-12^{\circ} \mathrm{C}$, con una humedad relativa promedio de $87 \%$. Los suelos son negros, húmedos, ácidos y con altos contenidos de materia orgánica, variando según la gradiente altitudinal: subpáramo con $\mathrm{pH}$ entre 3.814.33 y materia orgánica entre 8.6-12.4\% y páramo propiamente dicho con $\mathrm{pH}$ entre 4.45-4.83 y materia orgánica entre 20.5-26.8\% (Marcelo \& Millán, 2004).

\section{Metodología}

La evaluación se hizo a través de sondeos botánicos rápidos, por su velocidad y eficiencia en escalas amplias de diversos paisajes; sin embargo, se sacrifica información detallada en la estructura de la vegetación, pero no la de composición florística de 
especies, pues se elimina la interferencia inherente, al ignorar especies vistas en el ecosistema no presentes en una parcela (Vanclay, 1998).

Se realizaron cinco evaluaciones en diferentes épocas y años: Julio y Septiembre de 2002, Febrero y Octubre de 2003 y Julio de 2004, con la finalidad de colectar un mayor número de muestras botánicas fértiles. Las colectas se hicieron en caminos o trochas ya establecidas, tratando de abarcar todos los hábitats de la zona de estudio. De cada especie se colectó tres o cinco ejemplares duplicados.

Las colecciones botánicas fueron realizadas siguiendo los criterios descritas por Rodríguez \& Rojas (2002). La determinación se realizó en el Herbario CPUN (Universidad Nacional de Cajamarca), utilizando claves taxonómicas, comparando con especimenes existentes y revisando el material bibliográfico. Todo el material botánico fue depositado en los herbarios CPUN y MOL (Universidad Nacional Agraria La Molina).

Finalmente se hizo una lista de las especies encontradas ordenándolas según género y familia, siguiendo el orden alfabético y luego se comparó con la información de Tyron \& Stolze (1989) y Brako \& Zarucchi (1993).

\section{Resultados y discusión}

Se logró colectar 584 muestras botánicas que representan 252 taxa de plantas vasculares, identificándose a nivel de especie 151 plantas (60\%), 101 (40\%) a nivel de género y/o familia (Anexo 1).

Diversidad

Se reportan un total de 252 especies en 133 géneros pertenecientes a 58 familias. El 11.5\% (29 especies) corresponde a Pteridophyta, el 18.7\% (47 especies) a monocotiledóneas y el 69.8\% (176 especies) a dicotiledóneas. Tres son citas nuevas de géneros para el páramo del Neotrópico: Cacosmia, Paranephelius y Lomatia, así mismo, 40 especies son citas nuevas para la lista de plantas vasculares propuesta por Luteyn (1999). Anthericum, Palicuorea y Aphelandra, fueron registrados a $2900 \mathrm{msnm}$, mezclada con vegetación del bosque montano y el subpáramo; nuevos estudios podrían confirmar nuevos géneros en la zona.

En Pteridophyta se encontraron 29 especies de 14 géneros correspondientes a 8 familias, donde Polypodiaceae es la más diversa con 6 especies de 4 géneros, seguido de Pteridaceae con 6 especies de 3 géneros; Lycopodiaceae presenta 9 especies en 2 géneros (mayor diversidad de especies que las antes mencionadas); las demás familias están representadas con una sola especie.

En Angiospermas, Clase Monocotiledonae, se registraron 47 especies de 30 géneros correspondientes a 8 familias, siendo Orchidaceae la más diversa seguida de Poaceae y Liliaceae. La Clase Dicotiledonae está representada por 176 especies de
90 géneros correspondientes a 42 familias siendo la más diversa Asteraceae seguida de Ericaceae, Melastomataceae, Scrophulariaceae, Campanulaceae y Rosaceae.

Los géneros más diversos son: Senecio (8 especies), Miconia y Valeriana (7 especies cada uno), Calceolaria (6 especies), Arcytophyllum, Baccharis, y Huperzia (5 especies cada uno), Carex, Bomarea, Brachyotum, Lycopodium, Pleurothallis, Weinmmania y Monnina (4 especies cada uno).

El páramo en estudio presenta afinidades florísticas con los páramos de la Cordillera Oriental de Colombia, donde las familias más diversas son Asteraceae, Poaceae, Orchidaceae, Polypodiaceae, Scrophulariaceae, Cyperaceae, Ericaceae, Umbeliferae, Melastomataceae y Rosaceae (Cleff, 1981). Sin embargo para el páramo peruano, Liliaceae y Campanulaceae son más diversas que Cyperaceae, Umbeliferae y Rosaceae.

Cleff (1981) indica también que en dicho páramo existen unos 260 géneros, de los cuales 20 géneros son de pteridophytas (9 familias), 62 de monocotiledóneas (12 familias) y 178 de dicotiledóneas (52 familias). En la Cordillera Occidental es difícil tener una cifra global, pero en Tatamá se encontraron unos 115 géneros y en el páramo de Frontino 170 géneros con unas 215 especies (Sánchez, 1998). En el diagnóstico de la vegetación de los páramos del Parque Nacional Podocarpus del Ecuador, se registraron 221 especies, 93 géneros y 61 familias (Cabrera, 2001). Los resultados obtenidos en la "Evaluación Biológica: Santuario Nacional Tabaconas-Namballe”, realizado en los bosques montanos y el páramo, se reportan 238 especies de fanerógamas, con 91 especies de páramo (Baldeón, 2002). Según nuestros resultados el páramo en estudio presenta mayor diversidad que el páramo de las Lagunas Arreviatadas del Santuario Nacional Tabaconas-Namballe, que los páramos de Cajanuma del Parque Nacional Podocarpus del Ecuador, que el Páramo de Tatamá y el Frontino de la Cordillera Occidental de Colombia.

Sagástegui et al. (1999), indican que la diversidad de especies de plantas del Norte del Perú es muy alta debido al enorme rango de topografía y clima, para el departamento de Cajamarca: reporta 2699 especies, Amazonas 3474 especies y San Martín 3827 especies. En la lista de plantas vasculares publicada por Luteyn (1999), en el páramo del Norte Perú, se reportan 912 especies. Aunque nuestro estudio no representan un inventario de todo el páramo del Norte del Perú, sin embargo, las 252 especies estudiadas representan el 9.3\% del total de las especies reportadas para el departamento de Cajamarca y el 27.6\% del total de especies reportadas para los páramos del Perú. Esto nos revela que la zona de estudio es altamente diversa. Cabe mencionar que el número de especies hasta hoy registrados es preliminar, estimamos que en los años venideros se harán revisiones críticas de los géneros 
Senecio, Miconia, Valeriana, Calceolaria, entre otros, que seguramente alterarán las cifras aquí presentadas.

Los páramos del Neotrópico presentan 22 géneros endémicos (propios del ecosistema). Las especies endémicas ecuatorianas que están en el páramo son alrededor de 270; Gentianella (Gentianaceae), Epidendrum (Orchidaceae), Lysipomia (Campanulaceae), Draba (Brassicaceae) y Lepanthes (Orchidaceae), son los cinco géneros más ricos en especies endémicas ecuatorianas (León- Yañez, 2000). Haciendo una revisión de Brako \& Zarucchi (1993), Molau (1988) y bases de datos en línea como W3TROPICOS, se citan 28 especies endémicas, siendo la familia Asteraceae y Scrophulariaceae (la primera con 6 especies y la segunda con 4 especies) las que presentan mayores endemismos. Sin embargo, este número sin duda se incrementará cuando se culmine la determinación del material botánico.

El páramo del Perú es una de las áreas biogeográficas que está pobremente documentada y aún falta mucho por explorar, especialmente en los distritos de Chontalí, Colasay, Sallique (Jaén), Tabaconas y Namballe (San Ignacio) y en los páramos de Huancabamba (Piura), por lo tanto, se debe acelerar la ejecución de trabajos de investigación sobre aspectos botánicos, ornitológicos, herpetológicos, ecológicos, etnobotánicos, etc., que contribuyan al pleno conocimiento del ecosistema.

\section{Conclusiones}

1. Las 252 especies registradas representan el 9.3\% del total de las especies reportadas para el departamento de Cajamarca, y el $27.6 \%$ del total de especies reportadas para los páramos del Perú.

2. El Páramo de la provincia de Jaén presenta alta diversidad y endemismos, sin embargo, se encuentra seriamente amenazado por las quemas frecuentes, la ganadería extensiva y la extracción de elementos de la biodiversidad, por lo tanto, consideramos que toda la región del páramo de la provincia de Jaén y provincias aledañas que aún no se encuentran en un Área Natural Protegida debe de considerarse como área prioritaria de conservación.

\section{Agradecimientos}

Expresamos nuestro agradecimiento a los doctores James Luteyn y Benjamín Øllgaard por la determinación de especies de plantas. Al Ing. Segundo Vaca M. por el apoyo con parte de la logística; a los Sres. Abraham Cueva Ramos y Domingo Bermeo Tarrillo, quienes estuvieron siempre disponibles para colaborar en el trabajo de campo y a todos lo que hicieron posible el desarrollo del presente trabajo.

\section{Literatura citada}

Baldeón S. 2003. Evaluación de la Diversidad Biológica de plantas del Santuario Nacional Tabaconas-Namballe.
Edit, por Amanzo, J. Informe WWF- OPP: QM-91. Pág. 32:40

Brako L. \& Zarucchi J.L. 1993. Catalogo de las Angiospermas y Gimnospermas del Perú. Monographs in Sistematic Botany. Missouri Botanical Garden. Pág. 1286

Cabrera C. 2001. Categorías de amenaza de las plantas endémicas de los páramos del Parque Nacional Podocarpus. Fundación Ecuatoriana para la Investigación y Desarrollo de la Botánica (FUNBOTÁNICA). Boletín 9. Loja. Ecuador.

Cleff, A. M. 1981. The vegetation of the páramos of the Colombia Cordillera Oriental. Diss. Bot. 61:1-320 J. Cramer, Vaduz

INRENA. 1994. Evaluación de Recursos Naturales y Plan de Protección Medio ambiental. Proyecto Especial Jaén, San Ignacio, Bagua. Vol. I-II.

León-Yánez S. 2000. La flora de los páramos ecuatorianos. En: La biodiversidad de los páramos. Serie Páramo 7: 5-21. GTP/AbyaYala. Quito.

Luteyn J.L. 1999. Páramos, a checklist of plant diversity geographical distribution and botanical literature. Memoirs of the The New York Botanical Garden vol. 84, The New York Botanical Garden Press, New York.

Marcelo J.L. \& Millán J.F. 2004. Estudio de la diversidad florística del páramo, sectores: El Espino y Palambe. Distrito de Sallique. Provincia de Jaén. Tesis para optar el título de Ingeniero Forestal. Universidad Nacional de Cajamarca - Sección Jaén.

Molau U. 1988. Scrophulariaceae-Part I. Calceolarieae. Flora Neotropica Monographs 35:1-290.

Rodríguez R. E. \& Rojas G. R. 2002. El Herbario. Administración y manejo de colecciones botánicas. Editado por R. Vásquez M. Jardín Botánico de Missouri - PERÚ.

Rodríguez L. 1996. Diversidad Biológica del Perú. Zonas Prioritarias para la Conservación. PROYECTO FANPE GTZ-INRENA. Lima - Perú.

Sagástegui A. Dillon M. Sánchez I. Gonzáles S. \& Asencio P. 1999. Diversidad Florística del Norte del Perú. Tomo I. WWF \& Universidad Privada Antenor Orrego, Trujillo. Perú

Sánchez D. 1998. Estudio Florístico del Páramo de Frontino (Urrao-Antioquia). Revista Facultad de Agronomía. Medellín. Vol 51 (2). Pág:99-156

Tryon R. \& Stolze R. 1989a. Pteridophyta of Perú. Part I. Fieldeana Botany N. S. 20 1989b. Pteridophyta of Perú. Part II. Fieldeana Botany N. S. 22 1991. Pteridophyta of Perú. Part IV. Fieldeana Botany N. S. 27 1992. Pteridophyta of Perú. Part III. Fieldeana Botany N. S. 29 1993. Pteridophyta of Perú. Part V. Fieldeana Botany N. S. 32

Vanclay J.K. 1998. Towars more rigorous assennment of biodiversity. In: Bachmann, P., Kohl, M., Paivinen, R. (Eds.) Assessment of Biodiversity for Improved Forest Planing. Kluwer Academic Publishers, Dordrecht. pp: 211-232.

Weberbauer A. 1945 El Mundo Vegetal de los Andes Peruanos. Ministerio de Agricultura. Lima. 


\section{Apéndice 1}

\section{LISTA PRELIMINAR DE LA DIVERSIDAD FLORÍSTICA DEL PÁRAMO EN ESTUDIO}

\section{DIVISIÓN PTERIDOPHYTA}

1. BLECHNACEAE

Blechnum auratum (Fée) R. M. Tryon \& Stol. Blechnum loxense H.B.K.

2. DENNSTAEDTIACEAE

Pteridium aquilinum (L.) Kunth var. arachnoideum (Kaulf.) Brade

3. DRYOPTERIDACEAE Elaphoglossum engelii (Karst.) Christ. Elaphoglossum huacsaro (Ruiz) Christ.

4. GLEICHENIACEAE

Gleichenia revoluta H.B.K. Gleichenia simplex (Desv.) Hook

5. LOPHOSORIACEAE

Lophosoria cuadripinnata (Gmelin) C. Chr.

6. LYCOPODIACEAE

Huperzia capellatae (Herter) Holub Huperzia cf. macbridei (Herter) B. Olla. Huperzia weberbaueri (Nessel) Holub Huperzia brevifolia (Hook. \&\&Grez.) Holub Huperzia sp. 1 Lycopodium clavatum L. subsp clavatum Lycopodium jussiaei Desv. Ex Poiret Lycopodium thyoides (H.\&B. ex Willd.) Rothm. Lycopodium vestivum Desv. ex Poiret

7. POLYPODIACEAE

Campyloneurum amphostenon (Kunze ex Klotzsch) Fee

Grammitis sp.

Grammitis flaveliformis (Poiret) Morton** Grammitis moniliformi (Lag.Ex Sw.) Proc.** Niphidium crassifolium (L.) Lell.

Pleopeltis macrocarpa (L.) Willd

8. PTERIDACEAE

Adiantum poiretti Wikstr

Eriosorus cheilanthoides (Sw.) A. F. Tryon

Eriosorus elongatus (Hook. \& Grev.) Capel Jamesonia sp. (1679)

Jamesonia alstonii A. F. Tryon

Jamesonia goudotii (Hieron) C. Chr.

\section{DIVISIÓN ANGYOSPERMAE CLASE MONOCOTILEDONEAE}

1. BROMELIACEAE

Pitcairnia pungens H.B.K.

Puya fastuosa Mez. *

Puya sp.

Taxón desconocido 1651

Taxón desconocido 1652

Taxón desconocido 1658

2. CYPERACEAE

Bulbostylis juncoides (M. Vahl) Kuekenthal var. juncoides**

Bulbustylis sp.

Carex sp. 1

Carex sp. 2

Carex sp. 3

Carex pichinchensi Kunth

3. IRIDACEAE

Sisyrinchum trinerve Baker

4. LILIACEAE

Anthericum glaucum R\&P

Bomarea dulcis (Hooker) Beauverd Bomarea edulis (Hooker) Beauverd ** Bomarea purpurea (R. \&P.) Herbert Bomarea rosea (R. \& P.) Herbert ** Excremis coarctata (R. \& P.) Baker Isidrogalvia falcata R. \& P.

5. ORCHIDACEAE

Altensteina sp.

Buchtienia boliviensis Schlechter

Elleanthus aurantiacus (Lindley)

Reichenbach

Encyclia vespa (Vell. Conc.) Dressler Epidendrum rhombochilum L.O. Wil Epidendrum funkii Reichenbach f. ** Epidendrum secundum Jacquin** Odontoglossum sp.

Oncidium excavatum Lindley

Pachyphillum pasti Rchb. f.

Pleurothallis sp. 1

Pleurothallis sp. 2

Pleurothallis sp. 3

Pleurothallis sp. 4

Stelis sp.

Taxón desconocido 11673

6. POACEAE

Agrostis tolucensis H.B.K.

Calamagrostis sp.

Chusquea sp.

Cortaderia sp.

Neurolepis aristata (Munro) A. Hitch. Paspalum bompladianum Fluegge** 
Poa sp.

Poichium sp.

7. SMILACEAE

Smilax sp.

8. XIRIDACEAE

Xiris subulata R. \& P.

\section{CLASE DICOTILEDONEAE}

1. ACANTHACEAE

Aphelandra wurdackii Wasshausen

2. ACTINIDACEAE

Saurauia sp.

3. AMARYLLIDACEAE

Stenomesson miniatum (Herb.) Rav.*

4. APIACEAE

Eryngium humile Cavanilles

Taxón desconocido

5. ARALIACEAE

Schefflera sp. 1

Schefflera sp. 2

Oreopanax sp.

6. ASTERACEAE

Achyrocline sp. 1

Achyrocline sp. 2

Achyrocline alata (H.B.K.) DC.

Ageratina azangaroensis (Schultz-Bip. Ex weddell) King \& H. Rob.

Ageratina exsertovenosa (Klatt) King \& H.

Robinson

Baccharis sp. 1

Baccharis sp. 2

Baccharis genistelloides (Lam.) Person

Baccharis obtusifolia H.B.K.

Baccharis sinuata H.B.K.

Bidens andicola Kunth

Cacosmia rugosa H.B.K. ***

Chrysactinium sp.

Chrysactinium acaule (H.B.K.) Weddell

Chrysactinium hieracioides (H.B.K.) H.

Robinson \& Brettell

Coreopsis oblanceolata S.F. Blake*

Coreopsis piurana Sherff *

Diplostephium sp.

Dorobaea pimpinelifolia (Kunth) B, Nordenstan

Dorobaea laciniata B. Nord.\&Pruski**

Gynoxis sp.1

Gynoxis sp.2

Gynoxis calyculisolvens Cuatrecasas*

Hierasium sp. 1
Hierasium sp. 2

Hierasium peruanum Fries * (**)

Hypochaeris sp.

Jungia sp.

Loricaria leptothamna (Matt.) Cuat.*

Oritrophium peruvianum (Lam.) Cuat.

Oritrophium repens (H.B.K.) Cuat.

Paranephelius jelskii (Hieron) H.Rob.\& Brettel*

Paranephelius sp.2***

Pentacalia sp.1

Pentacalia andicola (Turcz.) Cuatr.

Pentacalia peruviana (Person) Cuat.

Senecio sp. 1

Senecio sp. 2

Senecio sp. 3

Senecio sp. 4

Senecio sp. 5

Senecio sp. 6

Senecio laricifolius H.B.K.

Senecio usgurensis Cuatrecasas*

Stevia cf. andina B.L. Rob. **

Werneria nuvigena H.B.K.

Taxón desconocido 11634

Taxón desconocido 11659

7. BEBERIDACEAE

Berberis lobbiana (C.K. Schneid.) C. K.

Schneid.**

8. BETULACEAE

Alnus acuminata H.B.K.

9. BORAGINACEAE

Moritzia lindenii (A.DC.) Benth. ex Gurke

10. CAMPANULACEAE

Centropogon macbridei Gleason*

Lobelia tenera H.B.K.

Siphocampylus jelskii A. Zahlbr.

Lysipomia sp.1

Lysipomia sp.2

11. CAPRIFOLIACEAE

Viburnum incarum Graebner**

12. CHLORANTACEAE

Hedyosmum racemosum (R.\& P.) G. Don. **

13. CLETHRACEAE

Clethra fimbriata H.B.K.

14. CLUSIACEAE

Clusia sp.1

Clusia sp.2

Clusia sp.3

Hypericum aciculare Kunth

Hypericum laricifolium Jussieu

Hypericum recurvum N. Robson* 
15. COLUMELLIACEAE

Columellia lucida Danguy \& Cherm..

16. CORIARIACEA

Coriaria ruscifolia L.

17. CUNONIACEAE

Weinmannia sp.

Weinmannia chryseis Diels* (**)

Weinmannia elliptica Kunth

Weinmannia laurina H.B.K. aff. **

18. ERICACEAE

Bejaria aestuans L.

Bejaria resinosa Mutis ex L.f.

Cavendishia bracteata (R.\&P. ex A. St. Hilaire)

Hoerold

Disterigma sp.

Disterigma empetrifolium (H.B.K.) Drude

Gaultheria erecta Ventenat

Gaultheria reticulata H.B.K.

Gaultheria vaccinioides Weddell

Macleania rupestris (H.B.K.) A.C. Smith

Pernettya prostrata (Cav.) Sleumer

Vaccinium sp.

Vaccinium crenatum (G. Don) Sleu.

Vaccinium floribundum H.B.K.

19. ERIOCAULACEAE

Paepalanthus sp.

20. FABACEAE

Lupinus sp. 1

Lupinus sp. 2

Lupinus sp. 3

21. GENTIANACEAE

Gentiana postrata Haenke **

Gentianella sp.

Gentianella sp.

Halenia bella Gilg * (**)

Halenia brevicornis (H.B.K.) G. Don**

22. GERANIACEAE

Geranium humboldtii Willd ex Spreng

Geranium piurensis Kunth* (**)

23. LAMIACEAE

Satureja guamaniensis Mansfeld*

Satureja pulchella (Kunth) Briquet

24. LENTIBULARIACEAE

Pinguicola involuta R. \& P

25. LORANTHACEAE

Aetanthus macranthus (Hooker) Kuijt**

Tristerix $(1005,1266)$
26. LYTRACEAE

Cuphea ciliata R. \& P.

27. MELASTOMATACEAE

Axinaea sp.1

Axinaea nitida Cogniaux**

Brachyotum sp. 1

Brachyotum sp. 2

Brachyotum sp. 3

Brachyotum naudinii Triana

Meriana sp. 1

Meriana sp. 2

Miconia sp. 1

Miconia sp. 2

Miconia sp. 3

Miconia sp. 4

Miconia centrophora Naudin aff.**

Miconia lutescens (Bonpland) DC* (**)

Miconia vacciniodes (Bonp.) Naud.*

Tibouchina laxa (Desrous.) Cogniaux**

28. MYRICACEAE

Myrica pubescens H. \& B. ex Wild.

29. MYRSINACEAE

Geissanthus sp.

Cybianthus sp. (**)

30. MYRTACEAE

Myrcianthes fimbriata (H.B.K.) Mc Vaugh* (**)

31. OXALIDACEAE

Oxalis sp.

32. PASSIFLORACEAE

Pasiflora sp. 1

Pasiflora sp. 2

33. PLANTAGINACEAE

Plantago australis Lamarck

34. PIPERACEAE

Peperomia galioides Kunth

Peperomia sp.

Piper sp.

35. POLYGALACEAE

Monnina sp.

Monnina conferta R. \& P.*

Monnina ligustrifolia (Bonpl.) Eriksen* (**)

Monnina salicifolia R. \& P.

36. PROTEACEAE

Lomatia hirsuta (Lam.) Diles ex Macb.***

Oreocallis grandiflora (Lamarck) R. Brown

37. ROSACEAE

Alchemilla verticilada Field. \& Gard.** 
Diciembre 2006

Hesperomeles sp.

Hesperomeles heterophylla (R.\&P.) Hook**

Rubus acanthophyllos Focke*

Rubus weberbaueri Focke*

Rubus sp.

38. RUBIACEAE

Arcytophyllum sp.

Arcytophyllum ciliolatum Standl.

Arcytophyllum ericoides (Willd. ex Roemer \&

Schultes) Standley

Arcytophyllum filiforme (R.\&P.) Stand.

Arcytophyllum setosum (R.\&P.) Stand.

Palicourea sp.

\section{SCROPHULARIACEAE}

Alonsoa sp.

Bartsia sp.

Bartsia crisafulli N. Holmgren subsp crisafulli

Bartsia mutica (Kunth) Bentham

Calceolaria sp. 1

Calceolaria sp. 2

Calceolaria gaultherioides Molau*

Calceolaria rhododendroides Kraenzlin*

Calceolaria tomentosa R. \& P.* (**)

Calceolaria trilobata Hemsley subsp.

Aequilateralis (Edwin) Molau*
Castilleja fissifolia L.

Taxón desconocido 1068, 1299

40. SOLANACEAE

Solanum sp.

41. VALERIANACEAE

Phyllactis rigida $\mathrm{R}$. \& P.

Valeriana sp. 1

Valeriana sp. 2

Valeriana sp. 3

Valeriana sp. 4

Valeriana sp. 5

Valeriana sp. 6

Valeriana punctata F. Meyer

42. VIOLACEAE

Viola dombeyana $\mathrm{DC}^{* *}$

* Especies endémicas.

**Nueva cita de especie para la lista de plantas vasculares del Neotrópico

***Nuevo cita de género para la lista de plantas vasculares del Neotrópico

* (**) Especie endémica y nueva cita de especie para la lista de plantas vasculares del Neotrópico

${ }^{1}$ Universidad Nacional Agraria La Molina. Herbario MOL. Facultad de Ciencias Forestales, Departamento Académico de Manejo Forestal, Lima Perú. E-mail: jolmarp2@yahoo.es.

${ }^{2}$ Universidad Nacional de Cajamarca, Herbario CPUN, Departamento de Biología. Apartado 55. Cajamarca Perú. E-mail: svisidoro@yahoo.com

${ }^{3}$ Universidad Nacional de Cajamarca, Departamento de Ciencias agrícolas y Forestales, Sección- Jaén. E-mail: josemt_777@hotmail.com 